\title{
Suppression of microRNA-96 expression inhibits the invasion of hepatocellular carcinoma cells
}

\author{
RONG-XIN CHEN, YUN-HONG XIA, TONG-CHUN XUE and SHENG-LONG YE \\ Liver Cancer Institute and Zhongshan Hospital, Fudan University, Key Laboratory of Carcinogenesis \\ and Cancer Invasion, Ministry of Education, Shanghai 200032, P.R. China
}

Received July 18, 2011; Accepted October 17, 2011

DOI: $10.3892 / \mathrm{mmr} .2011 .695$

\begin{abstract}
MicroRNA-96 (miR-96) expression is dysregulated in certain types of cancers. However, the role of miR-96 in hepatocellular carcinoma (HCC) invasion and metastasis remains elusive. miR-96 expression was investigated in a number of HCC cell lines by quantitative reverse transcription-polymerase chain reaction (RT-PCR). Cell invasive ability of metastatic HCCLM6 cells transfected with anti-miR-96 oligonucleotides or miR-96 mimic was determined by Matrigel invasion assay in vitro. In addition, metastasis-associated protein, osteopontin, was evaluated by Western blotting. miR-96 expression was significantly increased in highly metastatic HCCLM6 cells. Decreasing miR-96 expression with anti-miR-96 oligonucleotides led to reduced migration and invasion of HCCLM6 cells in vitro. In particular, down-regulation of miR-96 decreased osteopontin expression in HCCLM6 cells. Suppression of miR-96 expression inhibits the invasion of HCC cells, suggesting that miR-96 may be a therapeutic target for inhibiting HCC invasion and metastasis.
\end{abstract}

\section{Introduction}

Hepatocellular carcinoma (HCC) is one of the most aggressive types of cancer worldwide and is associated with high mortality and poor prognosis (1). Despite improvements in available treatments, such as surgical resection, liver transplantation, locoregional therapy and transcatheter arterial chemoembolization, HCC prognosis remains poor owing to the high rate of recurrence or metastasis following therapy $(2,3)$. Thus, it is critical to identify the molecules controlling the invasive and metastatic potential of $\mathrm{HCC}$, which may provide new targets for intervention.

Correspondence to: Dr Sheng-Long Ye, Liver Cancer Institute and Zhongshan Hospital, Fudan University, Key Laboratory of Carcinogenesis and Cancer Invasion, Ministry of Education, Shanghai 200032, P.R. China

E-mail: ye.shenglong@zs-hospital.sh.cn

Key words: hepatocellular carcinoma, microRNA-96, osteopontin
Recently, microRNAs (miRNAs), a new class of small noncoding RNAs, have been discovered and implicated as playing a key role in tumor development and progression of cancer (4). By binding to the complementary sequences of their target mRNAs, miRNAs induce mRNA degradation or translational repression. Dysregulation of miRNAs may affect oncogenes or tumor-suppressor genes, thereby having implications in cancer development and metastasis. miRNA-96 (miR-96) is aberrantly expressed in certain types of cancer (5-7). It has been reported that miR-96 is overexpressed in hepatitis B virus (HBV)-associated HCC (7). However, the role of miR-96 in HCC invasion and metastasis remains enigmatic.

To investigate the role of miR-96 in HCC invasion and metastasis, we used quantitative reverse transcription-polymerase chain reaction (RT-PCR) to compare the expression of miR-96 in a number of HCC cell lines with varying metastatic potential. Our data indicated that miR-96 expression was correlated with the metastatic potential of HCC cells and that miR-96 expression was significantly elevated in HCCLM6 cells with high metastatic potential. In addition, we also found that suppression of miR-96 expression with synthetic anti-miR-96 oligonucleotides inhibited migration and invasion of HCCLM6 cells in vitro. Down-regulation of miR-96 also decreased metastasis-associated protein osteopontin (OPN) expression in HCCLM6 cells. Therefore, these results suggest that miR-96 may be a potential target for inhibiting HCC invasion and metastasis.

\section{Materials and methods}

Cell culture. The SMMC-7721 human HCC cell line and the L02 normal liver cell line were obtained from the Shanghai Institute of Cell and Biology, Chinese Academy of Science, and maintained in RPMI supplemented with $10 \%$ fetal bovine serum (FBS) at $37^{\circ} \mathrm{C}$ with $5 \% \mathrm{CO}_{2}$. The MHCC97-L and HCCLM6 human metastatic HCC cell lines were established at the Liver Cancer Institute, Zhongshan Hospital, Fudan University, Shanghai, China, and cultured in Dulbecco's modified Eagle's medium (DMEM) (Invitrogen, Carlsbad, CA, USA) containing $10 \% \mathrm{FBS}$ at $37^{\circ} \mathrm{C}$ with $5 \% \mathrm{CO}_{2}$.

Transfection. Transient transfections of miRNA inhibitors and mimics were accomplished by Lipofectamine 2000 (Invitrogen). Inhibitor of miR-96 (sequence: 5'-GCAAAA 
Table I. miR-96 and U6 snRNA primers for quantitative RT-PCR .

\begin{tabular}{ll}
\hline Name & \multicolumn{1}{c}{ Sequence } \\
\hline miR-96 & \\
Sense & 5'-TTTGGCACTAGCACAT-3' \\
Antisense & 5'-GAGCAGGCTGGAGAA-3' \\
Probe & FAM-CGAGCGGCAAAAATCGCTCG-DABCYL \\
U6 snRNA & \\
Sense & 5'-ATTGGAACGATACAGAGAAGAT-3' \\
Antisense & 5'-GGAACGCTTCACGAATTT-3' \\
Probe & FAM-TGGCCCCTGCGCAAGGATG-DABCYL
\end{tabular}

snRNA, small nuclear RNA; miR-96, microRNA-96; RT-PCR, reverse transcription-polymerase chain reaction.

AUGUGCUAGUGCCAAA-3'), mimic of miR-96 (sequence: 5'-UUUGGCACUAGCACAUUUUUGC-3') and negative miR-control were purchased from GenePharma (Shanghai, China). HCC cells were seeded in 6-well plates and transfected with $4 \mathrm{nM}$ miR-96 inhibitor or $4 \mathrm{nM}$ miR-96 mimic.

RNA isolation and quantitative RT-PCR. Total RNA was extracted from cells using TRIzol reagent (Invitrogen). The expression of miR-96 was measured in HCC cell lines and in HCCLM6 cells transfected with miR-96 inhibitor or miR-96 mimic using the Hairpin-it miRNAs qPCR Quantitation Assay, according to the manufacturer's instructions (GenePharma). The primers used are listed in Table I. The U6 small nuclear RNA (U6 snRNA) was used as an internal control.

Western blotting. HCCLM6 cells were transfected in 6-well plates with the indicated concentrations of miR-96 inhibitor or miR-control. Following transfection, cells were cultured for $48 \mathrm{~h}$. Western blotting was used to assess OPN expression. Briefly, $20 \mu \mathrm{g}$ of cell lysate was separated by standard sodium dodecyl sulfate polyacrylamide gel electrophoresis (SDS-PAGE) and then transferred to polyvinylidene fluoride (PVDF) membranes. The membranes were washed, blocked and incubated with specific primary anti-human OPN monoclonal antibody (1:800) or an anti-glyceraldehyde 3-phosphate dehydrogenase (GADPH) antibody (1:5,000; Santa Cruz Biotechnology Inc., Santa Cruz, CA, USA), followed by incubation with horseradish peroxidase-conjugated secondary antibodies. The reactions were detected by enhanced chemiluminescence assay.

Cell proliferation assay. The cellular proliferation of transfected cells was measured by the MTT assay (Chemicon International Inc., Temecula, CA, USA). Cells $\left(1 \times 10^{4}\right)$ were plated in 96-well plates and incubated for 1-4 days, after which the cells were assayed at the indicated times following the manufacturer's instructions.

Matrigel invasion assay and migration assay. The invasive ability of the transfected cells was determined using Matrigel (BD Pharmingen, San Diego, CA, USA)-coated 24-well Transwell chambers, with upper and lower culture compart-

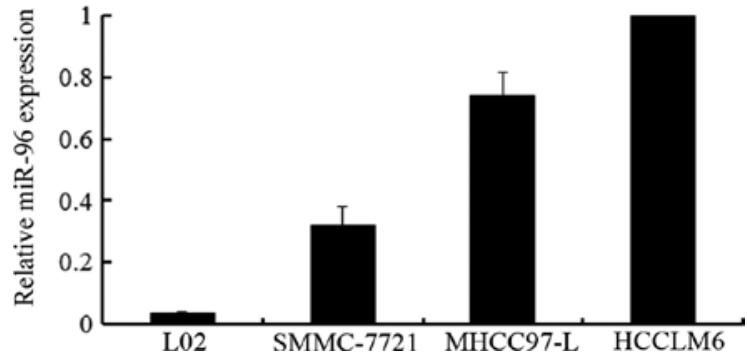

Figure 1. miR-96 expression was increased in HCCLM6 cells with high metastatic potential relative to other cell lines. miR-96 expression in HCC cell lines with varying metastatic potential was detected by quantitative RT-PCR. miR-96, microRNA-96; HCC, hepatocellular carcinoma; RT-PCR, reverse transcription-polymerase chain reaction.

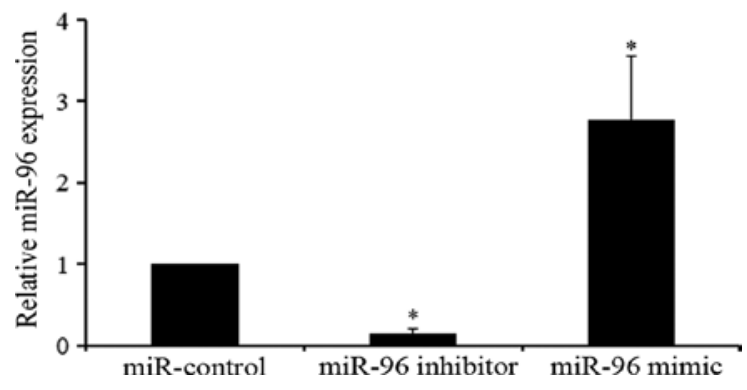

Figure 2. miR-96 expression in HCCLM6 cells transfected with miR-96 inhibitor or miR-96 mimic was greatly altered compared to cells transfected with miR-NC $($ ( $\mathrm{P}<0.05)$. miR-96 expression was measured by quantitative RT-PCR. miR-96, microRNA-96; RT-PCR, reverse transcription-polymerase chain reaction.

ments separated by polycarbonate membranes with $8-\mu \mathrm{m}$ pores (Corning, Costar, NY, USA). The bottom chamber was filled with DMEM containing $10 \%$ FBS as a chemoattractant. The transfected cells $\left(1 \times 10^{5}\right)$ were seeded on the top chamber and incubated at $37^{\circ} \mathrm{C}$ with $5 \% \mathrm{CO}_{2}$. After $40 \mathrm{~h}$, the cells were removed from the upper surface of the Matrigel by scrubbing with a cotton swab, and cells that migrated to the underside of the membrane were stained with Giemsa (Sigma). A total of 5 high-power fields were counted, and the mean number of cells per field was calculated. The migration assay protocol was similar to that of the invasion assay, but did not include the use of Matrigel. The experiments were performed in triplicate.

Statistical analysis. Data are reported as the means \pm standard deviation (SD) of three independent experiments, with each performed in triplicate. Statistical significance between treatment and control groups was analyzed using the Student's $\mathrm{t}$-test, and $\mathrm{P}<0.05$ indicated statistical significance.

\section{Results}

Expression of miR-96 in HCC cell lines with varying metastatic potential. To evaluate the relationship between miR-96 expression and metastatic potential, we applied quantitative RT-PCR to detect miR-96 expression in HCC cell lines with varying metastatic potential. The results revealed that miR-96 was expressed at a high level in HCCLM6 cells with high 
A

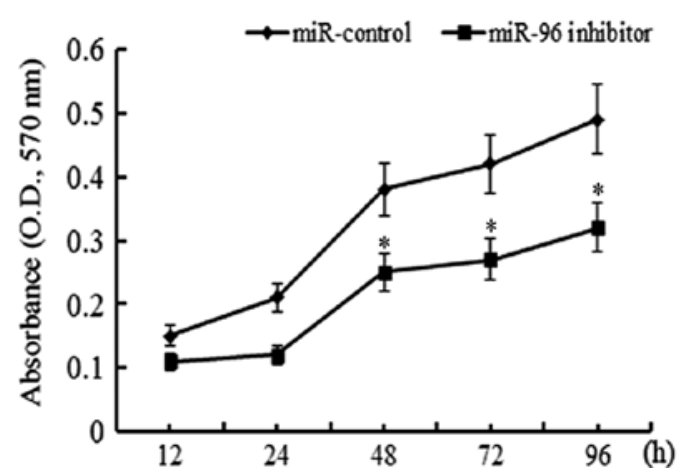

B

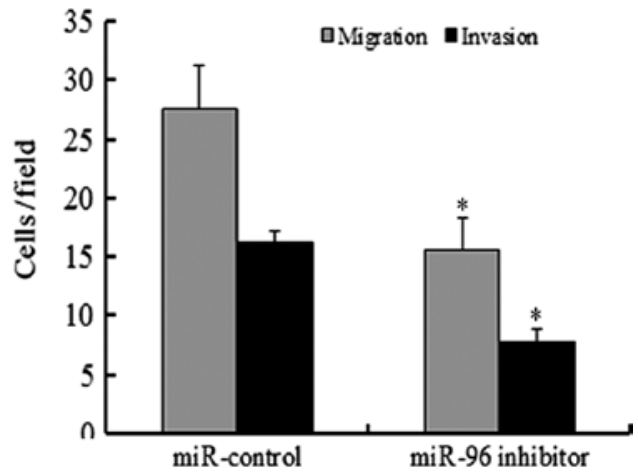

Figure 3. Effect of miR-96 expression on the proliferation, migration and invasion of HCCLM6 cells in vitro. (A) miR-96 inhibitor significantly suppressed cell proliferation of HCCLM6 compared to cells treated with miR-control (" $\mathrm{P}<0.05$ ). The proliferative rate of cells was assessed by the MTT assay. (B) miR-96 inhibitor significantly inhibited the migration and invasion of HCCLM6 cells compared to cells treated with miR-control (*P<0.05). The migratory and invasive capabilities of HCCLM6 cells were assessed using Transwell chambers. miR, microRNA; OD, optical density.

A



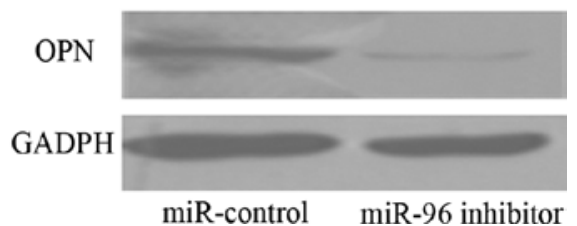

Figure 4. Effects of miR-96 expression on OPN expression in HCCLM6 cells. (A) OPN mRNA expression in HCCLM6 cells treated with the miR-96 inhibitor was significantly decreased compared to miR-control-treated cells ( $\left.{ }^{*} \mathrm{P}<0.05\right)$. (B) OPN protein expression in HCCLM6 cells transfected with the miR-96 inhibitor was significantly reduced compared to the miR-control-treated cells. miR, microRNA; OPN, osteopontin; GADPH, glyceraldehyde 3-phosphate dehydrogenase.

metastatic potential, at a lower level in MHCC97-L cells with low metastatic potential, and at a much lower level in non-metastatic HCC SMMC-7721 cells. miR-96 expression was barely detectable in L02 normal liver cells (Fig. 1). These findings provide evidence that miR-96 expression is correlated with the metastatic phenotype of HCC cells.

Effects of down-regulation of miR-96 expression on in vitro proliferation and invasion in HCCLM6 cells with high metastatic potential. miR-96 expression was significantly down-regulated or increased in HCCLM6 cells transfected with the miR-96 inhibitor or miR-96 mimic compared to miR-control-transfected cells (Fig. 2). To evaluate the effects of changes of the miR-96 level on the in vitro proliferation and invasion in HCCLM6 cells, functional assays were performed. Suppression of miR-96 expression significantly inhibited the proliferation of HCCLM6 cells $(\mathrm{P}<0.05)$ (Fig. 3A), suggesting that miR-96 may be involved in cell growth. As migratory and invasive behaviors are indicators of metastatic potential, we examined HCCLM6 migration and invasion in vitro using the Transwell assay, after miR-96 was down-regulated by the miR-96 inhibitor. The numbers of HCCLM6 cells transfected with miR-96 inhibitor that migrated toward the conditioned medium or invaded through the Matrigel were significantly lower than those of the miR-control-transfected cells (migration assay: $15.6 \pm 2.7$ vs. $27.6 \pm 3.6, \mathrm{P}<0.05$; invasion assay: $7.8 \pm 2.2$ vs. 16.2 $\pm 2.2, \mathrm{P}<0.05$ ) (Fig. 3B), suggesting that miR-96 contributes to the invasive and metastatic potential of HCCLM6 cells. However, increasing miR-96 expression by miR-96 mimic did not affect the in vitro proliferation and invasion of HCCLM6 cells compared to cells transfected with the miR-control (data not shown); this implies that miR-96 produced by tumor cells is sufficient for maintaining the malignant phenotype.

OPN expression was reduced following suppression of $\mathrm{miR}-96$ expression in HCCLM6 cells. After HCCLM6 cells were transfected with the miR-96 inhibitor, total RNA was reverse transcribed, and the relative amount of OPN mRNA was measured by real-time quantitative PCR analysis (Fig. 4A). In parallel, we evaluated the expression of OPN in HCCLM6 cells transfected with the miR-96 inhibitor. Following transfection with the miR-96 inhibitor for $48 \mathrm{~h}$, protein levels of OPN were significantly reduced compared to those in the miR-controltransfected cells (Fig. 4B), suggesting that OPN is involved in the response to decreased miR-96 expression.

miR-96 expression in HCC progression. To explore the role of miR-96 in HCC progression, we examined the expression 
A

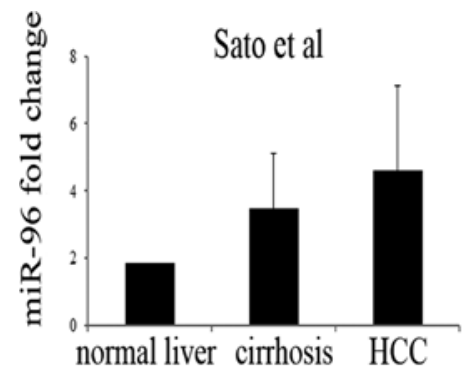

C



B

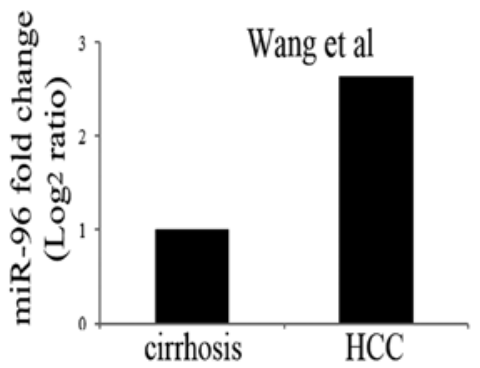

D

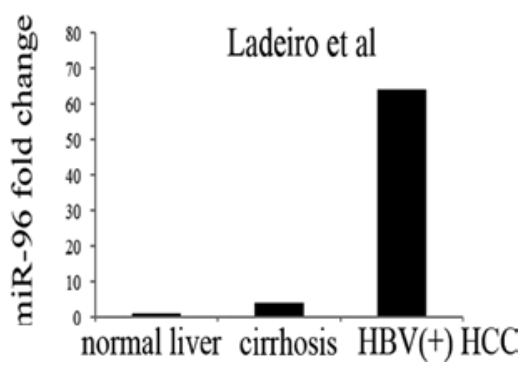

Figure 5. miR-96 was significantly up-regulated in hepatocellular carcinoma (HCC) tissues in four independent data sets: (A) Sato et al (8); (B) Wang et al (10); (C) Pineau et al (9); (D) Ladeiro et al (7). miR, microRNA.

profiles of miR-96 miRNA in HCC as reported by other authors (7-10). As shown in Fig. 5, miR-96 expression levels were gradually elevated from normal liver, non-tumorous liver tissues with chronic inflammation to HCC tissues. The highest level of miR-96 expression was observed in HCC in four independent data sets, suggesting that miR-96 plays a crucial role in HCC progression.

\section{Discussion}

miRNAs are small, non-coding, single-stranded RNAs that inhibit gene expression at the post-transcriptional level, and their abnormal expression has been described in various tumors (11-14). Although the number of verified human miRNAs is still expanding, only a few have been functionally described. However, with increasing understanding of the cellular behaviors affected by miRNA, modulating miRNA activities may provide exciting opportunities for cancer therapy (15-18). Here, we demonstrated that miR-96 expression was associated with the metastatic potential of HCC cells and that suppression of miR-96 expression inhibits the invasion of metastatic HCC cells, probably through down-regulation of OPN expression in vitro. Therefore, these data suggest that miR-96 is a potential candidate target for inhibiting HCC invasion and metastasis with miRNA-based cancer therapeutics.

Increased miR-96 expression has been reported in certain cancers, including chronic myeloid leukemia (19), breast (6), colorectal (5) and HCC (9). miR-96 is one of the most significantly deregulated miRNAs in colorectal cancer, and the miRNA expression profile could have relevance to the biological and clinical behavior of this disease (5). Up-regulation of miR-96 in prostate cancer is associated with cancer recurrence following radical prostatectomy (20). A total of 3 miRNAs, including miR-96, were observed to be highly expressed in MCF-7 breast cancer cells, in which the expression of the transcription factor FOXO1 was suppressed. Antisense inhibi- tors to each of these miRNAs led to a significant increase in FOXO1 expression and a decrease in cancer cell viability (6). Regarding HCC, with quantitative RT-PCR to detect miRNA expression in benign and malignant hepatocellular tumors, miR-96 was found to be overexpressed in HBV-associated HCC (7). Recently, Pineau et al (9) found that a set of 12 miRNAs, including miR-96, are linked to disease progression from cirrhosis to HCC. These findings suggest that miR-96 plays a crucial role in HCC progression and that modulating miR-96 expression may be a new modality for cancer therapy. However, these studies did not further evaluate the value and feasibility of inhibiting miR-96 to suppress HCC progression.

In the present study, down-regulation of miR-96 expression with the miR-96 inhibitor subsequently reduced cell proliferation and invasion of highly metastatic HCCLM6 cells in vitro, suggesting that miR-96 may be a candidate target for inhibiting HCC progression and metastasis. In response to down-regulation of miR-96 expression, OPN expression in HCCLM6 cells was reduced, suggesting that decreasing miR-96 expression inhibits the invasion of HCC cells, probably through the down-regulation of OPN expression. To the best of our knowledge, this is the first study to show that miR-96 expression is associated with OPN expression. OPN has significant implications for increasing cell proliferation, enhancing migration and extracellular matrix invasion in vitro $(21,22)$. OPN expression has been linked to tumor progression and metastasis in a variety of cancers and is associated with clinical stage, portending a poor prognosis (23-26). As for HCC, OPN has been identified as the primary gene overexpressed in metastatic HCC (27) and is closely associated with HCC metastasis $(28,29)$. It has been reported that increased miR-96 expression promotes tumor progression, possibly through targeting tumor-suppressor genes, including transcription factors CHES1 or FOXO1 $(5,6,30)$. Therefore, further research is required to clarify the mechanisms by which dysregulated miR-96 expression affects OPN expression in HCC invasion 
and metastasis. It may also be that miR-96 modulates OPN expression indirectly by targeting tumor-suppressor genes to regulate OPN expression.

In conclusion, these results demonstrate that suppressing miR-96 expression inhibits the invasion of highly metastatic HCC cells in vitro, suggesting that miR-96 may be a novel target for anti-HCC invasion and metastasis.

\section{Acknowledgements}

This study was supported by a China State Key Basic Research Program Grant (no. 2004CB518708).

\section{References}

1. Parkin DM, Bray F, Ferlay J and Pisani P: Global cancer statistics, 2002. CA Cancer J Clin 55: 74-108, 2005.

2. Tang ZY, Ye SL, Liu YK, et al: A decade's studies on metastasis of hepatocellular carcinoma. J Cancer Res Clin Oncol 130: 187-196, 2004.

3. Llovet JM, Burroughs A and Bruix J: Hepatocellular carcinoma. Lancet 362: 1907-1917, 2003.

4. Iorio MV and Croce CM: MicroRNAs in cancer: small molecules with a huge impact. J Clin Oncol 27: 5848-5856, 2009.

5. Bandres E, Cubedo E, Agirre X, et al: Identification by real-time PCR of 13 mature microRNAs differentially expressed in colorectal cancer and non-tumoral tissues. Mol Cancer 5: 29, 2006.

6. Guttilla IK and White BA: Coordinate regulation of FOXO1 by miR-27a, miR-96, and miR-182 in breast cancer cells. J Biol Chem 284: 23204-23216, 2009.

7. Ladeiro Y, Couchy G, Balabaud C, et al: MicroRNA profiling in hepatocellular tumors is associated with clinical features and oncogene/tumor suppressor gene mutations. Hepatology 47: 1955-1963, 2008.

8. Sato F, Hatano E, Kitamura K, et al: MicroRNA profile predicts recurrence after resection in patients with hepatocellular carcinoma within the Milan Criteria. PLoS One 6: e16435, 2011.

9. Pineau P, Volinia S, McJunkin K, et al: miR-221 overexpression contributes to liver tumorigenesis. Proc Natl Acad Sci USA 107: 264-269, 2010.

10. Wang Y, Lee AT, Ma JZ, et al: Profiling microRNA expression in hepatocellular carcinoma reveals microRNA-224 up-regulation and apoptosis inhibitor-5 as a microRNA-224-specific target. J Biol Chem 283: 13205-13215, 2008.

11. Sassen S, Miska EA and Caldas C: MicroRNA: implications for cancer. Virchows Arch 452: 1-10, 2008.

12. Yang L, Belaguli N and Berger DH: MicroRNA and colorectal cancer. World J Surg 33: 638-646, 2009.
13. Lynam-Lennon N, Maher SG and Reynolds JV: The roles of microRNA in cancer and apoptosis. Biol Rev Camb Philos Soc 84: 55-71, 2009.

14. Deng S, Calin GA, Croce CM, Coukos G and Zhang L: Mechanisms of microRNA deregulation in human cancer. Cell Cycle 7: 2643-2646, 2008.

15. Li C, Feng Y, Coukos G and Zhang L: Therapeutic microRNA strategies in human cancer. AAPS J 11: 747-757, 2009.

16. Mishra PJ and Merlino G: MicroRNA reexpression as differentiation therapy in cancer. J Clin Invest 119: 2119-2123, 2009.

17. Rossi JJ: New hope for a microRNA therapy for liver cancer. Cell 137: 990-992, 2009.

18. Wang V and Wu W: MicroRNA-based therapeutics for cancer. Biodrugs 23: 15-23, 2009.

19. Agirre X, Jimenez-Velasco A, San Jose-Eneriz E, et al: Down-regulation of hsa-miR-10a in chronic myeloid leukemia $\mathrm{CD} 34^{+}$cells increases USF2-mediated cell growth. Mol Cancer Res 6: 1830-1840, 2008

20. Schaefer A, Jung M, Mollenkopf HJ, et al: Diagnostic and prognostic implications of microRNA profiling in prostate carcinoma. Int J Cancer 126: 1166-1176, 2010.

21. Rittling SR and Chambers AF: Role of osteopontin in tumour progression. Br J Cancer 90: 1877-1881, 2004.

22. Takafuji V, Forgues M, Unsworth E, Goldsmith P and Wang XW: An osteopontin fragment is essential for tumor cell invasion in hepatocellular carcinoma. Oncogene 26: 6361-6371, 2007.

23. Coppola D, Szabo M, Boulware D, et al: Correlation of osteopontin protein expression and pathological stage across a wide variety of tumor histologies. Clin Cancer Res 10: 184-190, 2004.

24. Wai PY and Kuo PC: Osteopontin: regulation in tumor metastasis. Cancer Metastasis Rev 27: 103-118, 2008.

25. Rangaswami H, Bulbule A and Kundu GC: Osteopontin: role in cell signaling and cancer progression. Trends Cell Biol 16: 79-87, 2006.

26. Chen RX, Xia YH, Cui JF, Xue TC and Ye SL: Osteopontin, a single marker for predicting the prognosis of patients with tumor-node-metastasis stage I hepatocellular carcinoma after surgical resection. J Gastroenterol Hepatol 25: 1435-1442, 2010.

27. Ye QH, Qin LX, Forgues M, et al: Predicting hepatitis B virus-positive metastatic hepatocellular carcinomas using gene expression profiling and supervised machine learning. Nat Med 9: 416-423, 2003.

28. Zhao J, Dong L, Lu B, et al: Down-regulation of osteopontin suppresses growth and metastasis of hepatocellular carcinoma via induction of apoptosis. Gastroenterology 135: 956-968, 2008.

29. Sun BS, Dong QZ, Ye QH, et al: Lentiviral-mediated miRNA against osteopontin suppresses tumor growth and metastasis of human hepatocellular carcinoma. Hepatology 48: 1834-1842, 2008.

30. Myatt SS, Wang J, Monteiro LJ, et al: Definition of microRNAs that repress expression of the tumor-suppressor gene FOXO1 in endometrial cancer. Cancer Res 70: 367-377, 2010. 